\title{
The association of telomere length and serum 25-hydroxyvitamin D levels in US adults: the National Health and Nutrition Examination Survey
}

\author{
Mohsen Mazidi ${ }^{1,2}$, Erin D. Michos ${ }^{3,4}$, Maciej Banach ${ }^{5,6}$
}

\author{
${ }^{1}$ Institute of Genetics and Developmental Biology, International College, University \\ of Chinese Academy, Beijing, China \\ ${ }^{2}$ Key State Laboratory of Molecular Developmental Biology, Institute of Genetics \\ and Developmental Biology, Chinese Academy of Sciences, Chaoyang, Beijing, China \\ ${ }^{3}$ Ciccarone Center for the Prevention of Heart Disease, Division of Cardiology, \\ Johns Hopkins School of Medicine, Baltimore, Maryland, USA \\ ${ }^{4}$ Department of Epidemiology, Johns Hopkins Bloomberg School of Public Health, \\ Baltimore, Maryland, USA \\ ${ }^{5}$ Department of Hypertension, Chair of Nephrology and Hypertension, \\ Medical University of Lodz, Poland \\ ${ }^{6}$ Cardiovascular Research Centre, University of Zielona Gora, Zielona Gora, Poland
}

Submitted: 29 October 2016

Accepted: 14 November 2016

Arch Med Sci 2017; 13, 1: 61-65

DOI: $10.5114 /$ aoms.2017.64714

Copyright $\odot 2016$ Termedia \& Banach

\section{Abstract}

Introduction: Higher vitamin D levels and longer telomere length $(\mathrm{TL})$ have been associated with lower risk of several chronic diseases and all-cause mortality. However, direct relationships between 25-hydroxyvitamin D (25(OH)D) levels and TL are not well established. Vitamin D could influence TL through its anti-inflammatory properties. This study aimed to assess the relationship between vitamin D levels and TL in US adults.

Material and methods: Participants of the National Health and Nutrition Examination Survey (NHANES) with data available on 25(OH)D and TL measures from 2001 to 2002 were included. 25(OH)D levels were measured by the DiaSorin Radioimmunoassay. We used multivariable-adjusted linear regression models, accounting for the survey design and sample weights.

Results: Of the 4347 eligible participants, $47.0 \%(n=2045)$ were men. The mean age was 42.7 years overall, 49.2 years in men and 42.5 years in women $(p=0.060)$. After adjustment for age, race, marital status, education, and C-reactive protein, each $1 \mathrm{ng} / \mathrm{ml}$ higher $25(\mathrm{OH}) \mathrm{D}$ level was associated with a 0.045 ( $95 \%$ confidence interval (Cl): 0.032 to 0.059$)$ longer telomere-to-single copy gene (T/S) ratio. This was driven by a significant association in women $(0.054$ (0.043 to 0.064)) and in men (0.036 (0.020 to 0.052)). However, after we further adjusted for smoking, body mass index, and physical activity, no significant relation was found in the overall sample ( $\beta$ coefficient $-0.026,95 \% \mathrm{Cl}:-3.16$, $1.67)$, for men $(-0.016(-3.72,2.64))$, or for women $(-0.052(-6.85,2.26))$.

Conclusions: Our findings support a possible positive association between $25(\mathrm{OH}) \mathrm{D}$ levels and telomere length. The implications of this association deserve further investigation.

Key words: telomere length, 25(OH)D, aging.

\section{Introduction}

Telomeres are nucleoprotein "caps" formed of repetitive DNA sequences and specialized proteins at the ends of chromosomes [1]. They

\author{
Corresponding author: \\ Dr Mohsen Mazidi \\ Institute of Genetics \\ and Developmental \\ Biology \\ International College \\ University of \\ Chinese Academy \\ China \\ Phone: 13167518660 \\ E-mail: moshen@genetics. \\ ac.cn
}


protect chromosomes from deterioration and fusion during mitosis [1]. Telomeres are progressively eroded with successive rounds of cell divisions and by attrition, attributed to processes such as oxidative stress [2]. There is variation in telomere length $(\mathrm{TL})$ between persons of equal chronological age, due to differences in both genetic and environmental determinants [3-5].

Shorter leukocyte TL is associated with aging [6] and age-related diseases such as cardiovascular disease (CVD) [7], diabetes mellitus (DM) [8], hypertension (HTN) [9], and all-cause mortality [10]. Epidemiological studies have found associations of higher vitamin D status, as measured by circulating 25 -hydroxyvitamin $\mathrm{D}(25(\mathrm{OH}) \mathrm{D})$ concentrations, with a lower risk of all-cause mortality and several non-skeletal diseases including CVD [11, 12], although there are inconsistencies across studies [13]. Vitamin D status could plausibly affect the maintenance of TL directly or via effects on mechanisms including inflammation and/ or the rate of cell proliferation $[14,15]$. If higher vitamin D status limits the rate of telomere erosion in a causal fashion, $25(\mathrm{OH}) \mathrm{D}$ concentrations could represent a modifiable treatment target for maintenance of TL over the life course. Two large cross-sectional studies have studied $25(\mathrm{OH}) \mathrm{D}$ concentrations in relation to TL in populations of healthy women (both pre- and postmenopausal) [16]. Both studies reported that higher $25(\mathrm{OH}) \mathrm{D}$ levels were associated with longer TL. In another study of US radiology technologists, vitamin D deficiency was associated with shorter TL in whites but not blacks [17]. However, to our knowledge, no study has investigated this issue in a large population-based sample of both US adult men and women.

Therefore, the aim of this study was to determine the relationship between serum vitamin $D$ levels and TL in a cohort of adults representative of the general US population.

\section{Material and methods}

\section{Population}

The National Health and Nutrition Examination Surveys (NHANES) are ongoing repeated cross sectional surveys conducted by the US National Center for Health Statistics (NCHS). NHANES uses a multistage probability sampling strategy, which oversamples certain subgroups of the population, including blacks, Mexican-Americans, and those of lower socioeconomic status. The NCHS Research Ethics Review Board approved the NHANES protocol and consent was obtained from all participants. About 5000 subjects participate in NHANES each year, and the data are reported in 2-year cycles available for public use. Data collection on demographic, dietary, and behavioral information occurs through in-home administered questionnaires, while anthropometric and biomarker data are collected by trained staff using mobile examination units. The interview consists of questions on socio-demographic characteristics (age, gender, education, race/Hispanic origin, and health insurance) and questions on previously diagnosed medical conditions. More detailed information on the NHANES protocol is available elsewhere [18].

This study was based on analysis of data from the 2001-2002 NHANES cycle. We included participants aged 18 years and older who had available data on both $25(\mathrm{OH}) \mathrm{D}$ and TL measures. Adults with a history of diabetes, coronary heart disease, angina, myocardial infarction, stroke or congestive heart failure were excluded.

\section{Telomere measurements}

Briefly, aliquots of purified DNA, isolated from whole blood using the Puregene (D-50 K) kit protocol (Gentra Systems, Inc., Minneapolis, Minnesota), were obtained from participants. The leukocyte telomere length (LTL) assay was performed in the laboratory of Dr. Elizabeth Blackburn at the University of California, San Francisco, using the quantitative polymerase chain reaction method to measure LTL relative to standard reference DNA (also known as the telomere-to-single copy gene (T/S) ratio) [19]. Each sample was assayed 3 times on 3 different days. The samples were assayed on duplicate wells, resulting in 6 data points. Control DNA values were used to normalize between-run variability. Runs with more than 4 control DNA values falling outside 2.5 standard deviations from the mean for all assay runs were excluded from further analysis (6\% of runs). For each sample, any potential outliers were identified and excluded from the calculations (2\% of samples). The Centers for Disease Control (CDC) conducted a quality control review before linking the LTL data to the NHANES data files. The CDC Institutional Review Board granted human subject approval for this study.

\section{Serum 25-hydroxyvitamin D}

Total serum 25(OH)D was measured at the $\mathrm{Na}$ tional Center for Environmental Health, CDC, Atlanta, GA, USA using a radioimmunoassay (RIA) kit (DiaSorin, Stillwater, MN, USA) [20]. The sensitivity of this assay has been shown to be $1.5 \mathrm{ng} / \mathrm{ml}$ and the coefficient of variance (CV) was 7\% [21].

\section{Statistical analysis}

We conducted the analyses according to the CDC guidelines for analysis of complex NHANES data, accounting for the masked variance and using the 
proposed weighting methodology [22]. We computed seasonal-adjusted serum 25(OH)D levels using analysis of covariance (ANCOVA). 25(OH)D was adjusted by season of examination. Continuous levels of $25(\mathrm{OH}) \mathrm{D}$ per $1 \mathrm{ng} / \mathrm{ml}$ higher value were used for our analysis.

To determine any association between serum vitamin $\mathrm{D}$ and $\mathrm{TL}$, we used progressively adjusted multivariable linear regression models. Our primary model adjusted for sex, race, education, marital status, and C-reactive protein (CRP). In a second model, we additionally adjusted for body mass index (BMI), smoking, and physical activity. Groups were compared using analysis of variance (ANOVA) and $\chi^{2}$ tests. All tests were two sided, and $p<0.05$ was the level of significance. Results were analyzed using SPSS complex sample module version 22.0 (IBM Corp, Armonk, NY). Sample weights were applied to account for unequal probabilities of selection, nonresponse bias, and oversampling.

\section{Results}

Of the 4347 eligible participants, $47.0 \%$ ( $n=$ 2045) were men. The mean age was 42.7 years overall, 49.2 years in men and 42.5 years in women $(p=0.060)$. With regard to education, $48.9 \%$ ( $n=1879)$ of the participants had completed more than high school, $23.6 \%(n=910)$ had completed high school, while $27.5 \%(n=1055)$ had completed less than high school.

Whites (non-Hispanic) represented 50.5\% ( $n=2196)$ of the participants, blacks represented $18.9 \%(n=820)$ and Mexican-Americans represented $23.0 \%(n=1001)$. With regard to marital status, $52.3 \%(n=2275)$ were married, $6.4 \%(n=277)$ were widowed and 6.9\% $(n=301)$ divorced. Mean and standard deviation of the $25(\mathrm{OH}) \mathrm{D}$ were for the overall population $23.3 \pm 8.8 \mathrm{ng} / \mathrm{ml}$, for men $23.4 \pm 8.0 \mathrm{ng} / \mathrm{ml}$ and for women $23.3 \pm 9.4 \mathrm{ng} / \mathrm{ml}$. A total of $38.2 \%$ of the population had $25(\mathrm{OH}) \mathrm{D}$ deficiency (defined as 25(OH)D levels $<20 \mathrm{ng} / \mathrm{ml}$ ). Mean BMI was $27.5 \pm 6.1 \mathrm{~kg} / \mathrm{m}^{2}$ overall, $27.1 \pm 5.4$ $\mathrm{kg} / \mathrm{m}^{2}$ in men and $27.8 \pm 6.6 \mathrm{~kg} / \mathrm{m}^{2}$ in women.
In total, $20.4 \%$ were current smokers including $24.6 \%$ of men and $16.6 \%$ of women.

Table I shows the seasonal adjusted mean vitamin $D$ by quartiles of telomere length. There was no significant trend in mean $25(\mathrm{OH}) \mathrm{D}$ across quartiles of the TL (all $p>0.05$ ). In linear regression models adjusted for age, race, marital status, education, and CRP, overall and stratified by sex, we found a statistically significant association between vitamin $\mathrm{D}$ and $\mathrm{TL}$ in the overall sample ( $\beta$ coefficient $0.045(95 \% \mathrm{Cl}: 0.032$ to 0.059$)$ ), driven by a positive and significant association in women (0.054 (0.043 to 0.064)), and in men (0.036 (0.020 to 0.052)), with no evidence of statistical interaction by sex (interaction $p=0.231$ ). However, after we further adjusted for the previous covariates (age, race, marital status, education, and (RP) plus smoking, BMI and physical activity, no significant association was found in the overall sample $(-0.026(-3.16,1.67))$, in men $(-0.016$ $(-3.72,2.64))$, or in women $(-0.052(-6.85,-2.26))$.

\section{Discussion}

In this study we aimed to determine the relationship between serum 25(OH)D and TL in US adults. To our knowledge this study is the largest population-based study to examine this issue in adults across a broad age range (age: 18-80) who were free of any history of diabetes, coronary heart disease, angina, myocardial infarction, stroke or congestive heart failure, and in both men and women. Our results revealed an association between 25(OH)D levels and TL in limited-adjusted models, suggesting a possible role of $25(\mathrm{OH}) \mathrm{D}$ status in the maintenance of TL.

Our study findings are in line with several other published studies [16, 23]. In a study of 2,160 adult twin women in the United Kingdom, 25(OH)D levels were positively associated with TL [23]. In a study of 1,337 registered female nurses in the United States, Liu et al. [16] also reported a positive association of $25(\mathrm{OH}) \mathrm{D}$ with TL. However, in contrast, there have been several studies that

Table I. Seasonal-adjusted mean of serum vitamin D levels across quartiles of telomere length stratified by sex

\begin{tabular}{|c|c|c|c|c|c|c|}
\hline \multirow{2}{*}{\multicolumn{2}{|c|}{ Variables }} & \multicolumn{4}{|c|}{ Quarters of telomere length } & \multirow[t]{2}{*}{$P$-value } \\
\hline & & Q1 & Q2 & Q3 & Q4 & \\
\hline \multicolumn{2}{|l|}{$N$} & 878 & 888 & 890 & 876 & \\
\hline \multicolumn{2}{|c|}{$\mathrm{TL}$, mean $\pm \mathrm{SE}$ (T/S ratio) } & $0.78 \pm 0.08$ & $0.97 \pm 0.04$ & $1.12 \pm 0.05$ & $1.43 \pm 0.21$ & \\
\hline \multirow[t]{3}{*}{$25(\mathrm{OH}) \mathrm{D}[\mathrm{ng} / \mathrm{ml}]$} & Total $(n=4987)$ & $23.1 \pm 0.2$ & $27.3 \pm 0.31$ & $26.1 \pm 2.8$ & $25.7 \pm 2.0$ & 0.892 \\
\hline & Men $(n=2319)$ & $23.5 \pm 0.32$ & $25.8 \pm 0.11$ & $23.7 \pm 0.39$ & $23.8 \pm 0.41$ & 0.771 \\
\hline & Women $(n=2668)$ & $23.1 \pm 0.4$ & $26.1 \pm 2.1$ & $25.8 \pm 2.2$ & $25.5 \pm 2.2$ & 0.898 \\
\hline
\end{tabular}

ap-values for linear trend across quartiles of telomere length. Variables were compared across quartiles of telomere length using analysis of variance (ANOVA) test. 
have failed to find any association between 25(OH)D and TL. In a recent study by Liu et al. [17] among 1154 US radiologic technologists aged 4893 years old, little evidence was found in support of the association of vitamin D and TL. This could be attributed to the older age of the participants (mean age: 63.2), in comparison with our studies and others that have reported significant associations. However, no associations of 25(OH)D and LTL were found in a sample of male health professionals [24] or in another study of young adults (at age 31) from Finland [25]. Thus, the role of vitamin $D$ in TL remains inconclusive.

There are potential mechanisms that could explain an association between $\mathrm{TL}$ and vitamin $\mathrm{D}$ concentrations. Activated vitamin D decreases the mediators of systemic inflammation, such as interleukin-2 and tumor necrosis factor- $\alpha$ [26]. Vitamin $D$ receptors are ubiquitously expressed on $T$ and B lymphocytes, natural killer cells, and monocytes $[27,28]$, and through the down-regulation of cytokines and other pro-inflammatory factors, activated vitamin D exerts anti-inflammatory and anti-proliferative actions, which would affect the turnover rate of leukocytes [29]. It follows that vitamin D would attenuate the rate of TL attrition. Inflammation and oxidative stress are key determinants in the biology of aging [30], and TL dynamics appear to be influenced by the accruing burden of these characteristics [31].

It has been suggested that TL may serve as a cumulative index of an individual's lifelong burden of oxidative stress and inflammation [31]. Some of the factors that heighten oxidative stress and inflammation are genetic, but others are clearly environmental in nature, and a few may be easily modifiable. For instance, cigarette smoking [5, 32], obesity [5], and sedentary lifestyle [4] are associated with shortened TL. Whereas these lifestyle habits may be difficult to change, vitamin $D$ concentrations are easily modifiable through nutritional supplementation or sunshine exposure. Our results found that after further adjustment for smoking, BMI and physical activity, there was no longer a statistically significant association between TL and 25(OH)D.

The findings from our study have to considered in the context of some study limitations. The cross-sectional nature does not allow inference about causality; it is possible there is reverse causation where individuals in poorer health do less outdoor physical activity and thus have lower 25(OH)D levels. Unfortunately, we did not have available any repeated measure of TL with quantitative polymerase chain reaction in the same subjects after several follow-up years to elucidate temporality of these findings.

The clinical relevance of the difference in relative $T L$ is also not known. The age of the subjects could have influenced the results, even though the data were adjusted for age. The RIA was used for this NHANES survey, but it is not the current gold standard of liquid chromatography-mass spectrometry (LC-MS). Moreover, 25(OH)D was measured at one time point, which may not reflect the lifetime vitamin D status, and there might be unmeasured confounders. NHANES measured 25(OH)D levels, which are felt to be the best marker of vitamin D stores, but it is activated vitamin $\mathrm{D}$ (or calcitriol) that binds to the vitamin $\mathrm{D}$ receptor and is thought to confer all the biological health effects. More studies are needed to replicate the results.

Understanding the interplay between vitamin D and $\mathrm{TL}$ is a necessary and important step toward any application of the resulting knowledge for public health policy and action. Our study provides a comprehensive snapshot of the relationship of 25(OH)D with TL at the national level in the US.

In conclusion, our findings support a possible positive association between $25(\mathrm{OH}) \mathrm{D}$ levels and telomere length; however, our results indicate that sedentary lifestyle and obesity may be crucial factors in any associations between 25(OH)D and TL. The implications of this association deserve further investigation.

\section{Conflict of interest}

The authors declare no conflict of interest.

\section{References}

1. Blackburn EH. Structure and function of telomeres. Nature 1991; 350: 569-73.

2. Kurz DJ, Decary S, Hong Y, Trivier E, Akhmedov A, Erusalimsky JD. Chronic oxidative stress compromises telomere integrity and accelerates the onset of senescence in human endothelial cells. J Cell Sci 2004; 117: 2417-26.

3. Codd V, Nelson CP, Albrecht E, et al. Identification of seven loci affecting mean telomere length and their association with disease. Nat Genet 2013; 45: 422-7, 7e1-2.

4. Cherkas LF, Hunkin JL, Kato BS, et al. The association between physical activity in leisure time and leukocyte telomere length. Arch Intern Med 2008; 168: 154-8.

5. Valdes AM, Andrew T, Gardner JP, et al. Obesity, cigarette smoking, and telomere length in women. Lancet 2005; 366: $662-4$.

6. Aviv A. Telomeres and human aging: facts and fibs. Sci Aging Knowledge Environ 2004; 2004: pe43.

7. Fitzpatrick AL, Kronmal RA, Gardner JP, et al. Leukocyte telomere length and cardiovascular disease in the cardiovascular health study. Am J Epidemiol 2007; 165: 14-21.

8. Salpea KD, Talmud PJ, Cooper JA, et al. Association of telomere length with type 2 diabetes, oxidative stress and UCP2 gene variation. Atherosclerosis 2010; 209: 42-50.

9. Vasan RS, Demissie S, Kimura M, et al. Association of leukocyte telomere length with circulating biomarkers of the renin-angiotensin-aldosterone system: the Framingham Heart Study. Circulation 2008; 117: 1138-44. 
10. Cawthon RM, Smith KR, O'Brien E, Sivatchenko A, Kerber RA. Association between telomere length in blood and mortality in people aged 60 years or older. Lancet 2003; 361: 393-5.

11. Dobnig H, Pilz S, Scharnagl H, et al. Independent association of low serum 25-hydroxyvitamin D and 1,25-dihydroxyvitamin D levels with all-cause and cardiovascular mortality. Arch Intern Med 2008; 168: 1340-9.

12. Kilkkinen A, Knekt P, Aro A, et al. Vitamin D status and the risk of cardiovascular disease death. Am J Epidemiol 2009; 170: 1032-9.

13. Shapses SA, Manson JE. Vitamin D and prevention of cardiovascular disease and diabetes: why the evidence falls short. JAMA 2011; 305: 2565-6.

14. Provvedini DM, Tsoukas CD, Deftos LJ, Manolagas SC. 1,25-dihydroxyvitamin D3 receptors in human leukocytes. Science 1983; 221: 1181-3.

15. Zhu H, Guo D, Li K, et al. Increased telomerase activity and vitamin $D$ supplementation in overweight African Americans. Int J Obes (Lond) 2012; 36: 805-9.

16. Liu JJ, Prescott J, Giovannucci E, et al. Plasma vitamin D biomarkers and leukocyte telomere length. Am J Epidemiol 2013; 177: 1411-7.

17. Liu JJ, Cahoon EK, Linet MS, et al. Relationship between plasma 25-hydroxymitamin D and leucocyte telomere length by sex and race in a US study. Br J Nutr 2016; 116: 953-60.

18. National Center for Health Statistics. National Health and Nutrition Examination Survey (NHANES). Questionnaires, datasets, and related documentation. www. cdc.gov/nchs/nhanes/nhanes questionnaires.htm. Accessed January 27, 2015.

19. Needham BL, Adler N, Gregorich S, et al. Socioeconomic status, health behavior, and leukocyte telomere length in the National Health and Nutrition Examination Sur vey, 1999-2002. Soc Sci Med 2013; 85: 1-8.

20. Centers for Disease Control and Prevention NC, Nutrition fHSndNHa, Survey. E. http://www.cdc.gov/nchs/ nhanes/ (accessed October 2015).

21. Nutritional Biochemistry Branch DoLS, National Center for Environmental Health (n.d.) Laboratory Procedure Manual 25-Hydroxyvitamin D 2001-2002.

22. Klein RJ, Schoenborn CA. Age Adjustment Using the 2000 Projected U.S. Population. Healthy People Statistical Notes, no 20. Hyattsville, Maryland: National Center for Health Statistics. January 2001.

23. Richards JB, Valdes AM, Gardner JP, et al. Higher serum vitamin D concentrations are associated with longer leukocyte telomere length in women. Am J Clin Nutr 2007; 86: 1420-5.

24. Julin B, Shui IM, Prescott J, Giovannucci EL, De Vivo I. Plasma vitamin D biomarkers and leukocyte telomere length in men. Eur J Nutr 2015 in press.

25. Williams DM, Palaniswamy S, Sebert S, et al. 25-Hydroxyvitamin D concentration and leukocyte telomere length in young adults: findings from the Northern Finland Birth Cohort 1966. Am J Epidemiol 2016; 183: 191-8.

26. Lemire JM. Immunomodulatory role of 1,25-dihydroxyvitamin D3. J Cell Biochem 1992; 49: 26-31.

27. Deluca HF, Cantorna MT. Vitamin D: its role and uses in immunology. FASEB J 2001; 15: 2579-85.

28. Mathieu C, Adorini L. The coming of age of 1,25-dihydroxyvitamin $\mathrm{D}(3)$ analogs as immunomodulatory agents. Trends Mol Med 2002; 8: 174-9.

29. Nagpal S, Na S, Rathnachalam R. Noncalcemic actions of vitamin D receptor ligands. Endocr Rev 2005; 26: 662-87.
30. Finkel T, Holbrook NJ. Oxidants, oxidative stress and the biology of ageing. Nature 2000; 408: 239-47.

31. Aviv A. Telomeres and human somatic fitness. J Gerontol A Biol Sci Med Sci 2006; 61: 871-3.

32. Nawrot TS, Staessen JA, Gardner JP, Aviv A. Telomere length and possible link to X chromosome. Lancet 2004; 363: 507-10. 